\title{
Retraction Note to: Detection of oasis soil composition and analysis of environmental parameters based on hyperspectral image and GIS
}

\author{
Yingfeng Chen ${ }^{1,2} \cdot$ Zhongyong Xiao $^{1,2} \cdot$ Guoqiang Chen ${ }^{1,2} \cdot$ Weimin $\mathrm{Su}^{2}$. Jiongfeng Chen ${ }^{1,2}$
}

Published online: 1 December 2021

(c) Saudi Society for Geosciences 2021

Retraction Note to: Arabian Journal of Geosciences (2021) 14: 1050 https://doi.org/10.1007/s12517-021-07407-6

The Editor-in-Chief and the Publisher have retracted this article because the content of this article is nonsensical. The peer review process was not carried out in accordance with the Publisher's peer review policy. Authors Yingfeng Chen, Zhongyong Xiao, Guoqiang Chen and Weimin Su have not responded to correspondence regarding this retraction. The Publisher has not been able to obtain a current email address for author Jiongfeng Chen.

The original article can be found online at https://doi.org/10.1007/ s12517-021-07407-6.

Yingfeng Chen

cyf88699@163.com

1 College of Harbour and Environmental Engineering, Jimei University, Xiamen 361021, Fujian, China

2 National Geographic Conditions Monitoring Research Center, Jimei University, Xiamen 361021, Fujian, China 